\title{
Do platelet mediators and coagulation factors affect the formation of neutrophil extracellular traps?
}

\author{
Iraklis C. Moschonas, Styliani Papadaki, Sofia Sidiropoulou, Alexandros D. Tselepis
}

Atherothrombosis Research Centre/Laboratory of Biochemistry, Department of Chemistry, University of loannina, loannina, Greece

\begin{abstract}
Aim: To investigate the effect of factor Xa (FXa), thrombin and platelets, under activation or resting conditions, on neutrophil extracellular traps (NETs) formation (NETosis). Materials and Methods: Neutrophils, isolated from apparently healthy volunteers, were challenged with 25-50 nM FXa, 0.2-8 U/mL thrombin, 5-100 $\mu \mathrm{M}$ adenosine diphosphate (ADP), 0.1-0.5 mM arachidonic acid (AA), 5-15 $\mu \mathrm{g} / \mathrm{mL}$ collagen, $100 \mu \mathrm{M}$ protease-activated receptor (PAR)-4 activating peptide, 10-100 $\mu$ M PAR-2 activating peptide or 25-100 nM phorbol 12-myristate 13-acetate (PMA). Moreover, neutrophils were challenged with platelet-rich plasma (PRP), activated with ADP (at a concentration of either $20 \mu \mathrm{M}$, in an aggregometer, or of $50 \mu \mathrm{M}$, in situ). Neutrophils were incubated for 3.5 h at $37^{\circ} \mathrm{C}$ and $5 \% \mathrm{CO}_{2}$ and an immunofluorescence protocol followed, in order to stain neutrophils and NETs for myeloperoxidase and DNA. Finally, NETs and neutrophils were microscopically visualized and the percentage of NET-releasing neutrophils of each of the above experimental conditions was evaluated. Results and Conclusion: Among the agonists studied, only PMA and AA, induced robust NETosis, at a threshold concentration of $25 \mathrm{nM}$ and $0.25 \mathrm{mM}$, respectively. Furthermore, platelets pre-activated with ADP, but not resting platelets induce the formation of NETs, a phenomenon that is also observed when platelets were activated with ADP in situ, in the presence of neutrophils. The present results provide an additional mechanism through which platelets contribute to various pathophysiological conditions in which NETs are implicated, such as inflammation and thrombosis. The whole spectrum of platelet mediators that are implicated in NETosis needs to be further investigated.
\end{abstract}

KEY WORDS: Arachidonic acid, factor Xa, neutrophil extracellular traps, platelets, thrombin

\section{INTRODUCTION}

Neutrophil extracellular traps (NETs) are threads of decondensed chromatin, which are decorated with numerous

\section{Corresponding author:}

Alexandros D. Tselepis, MD, PhD, Professor of BiochemistryClinical Chemistry, Atherothrombosis Research Centre/ Laboratory of Biochemistry, Department of Chemistry, University of loannina, 45110, loannina, Greece Tel.: +3026510 08365, Fax: +30265108785

E-mail: atselep@uoi.gr proteins and are generated by activated neutrophils. ${ }^{1}$ The procedure of the formation of NETs is termed as NETosis. Originally, NETs were studied for their anti-microbial properties, since they entrap and possibly kill pathogens, by providing a high local concentration of microbicidal proteases, such as neutrophil elastase and myeloperoxidase (MPO). ${ }^{1}$ However, later, extended research, has revealed that NETs are also present under sterile inflammatory

Submission: 05.06.2020, Acceptance: 15.06.2020 
conditions and they participate in the pathogenesis of multiple diseases, e.g. cancer, ${ }^{2}$ diabetes mellitus ${ }^{3}$ and Alzheimer's disease. ${ }^{4}$ Importantly, it is established that NETs are heavily involved in atherogenesis, as well as in arterial and venous thrombosis. ${ }^{5}$

In addition to their central role in the coagulation cascade, coagulation factors Xa (FXa) and thrombin exert cellular effects via protease-activated receptors (PARs), ${ }^{6,7}$ which are expressed on virtually all cell types. FXa activates PAR-1 and -2, whereas thrombin activates PAR-1, -3 and -4 , but not PAR-2.,

Platelets play a key role in hemostasis and thrombosis, however they also interact and activate leukocytes, including neutrophils, thus participating in inflammatory responses as well as in atherogenesis. ${ }^{8}$ The platelet-neutrophil interaction is mediated by P-selectin, expressed on the platelet surface, and P-selectin glycoprotein ligand-1, which is expressed on the surface of neutrophils. ${ }^{8}$

In the present study, we investigated the effect of FXa and thrombin, as well as platelets, in their resting and activation conditions, on neutrophils activation and NETs production.

\section{MATERIALS AND METHODS}

\subsection{Reagents}

Adenosine diphosphate (ADP), arachidonic acid (AA), thrombin from human plasma, Histopaque ${ }^{\circledR}-1119$, heat inactivated $(\mathrm{HI})$ human serum from clotted whole blood, phorbol 12-myristate 13-acetate (PMA) and 4',6-diamidino2-phenylindole (DAPI) were purchased from Sigma-Aldrich (St. Louis, MO, USA). Collagen type IV was purchased from Chrono-Log Corporation (Philadelphia, PA, USA). PAR-2 and -4 activating peptides were purchased from Bachem (Basel, Switzerland). FXa was purchased from Haematologic Technologies, Inc. (Essex Junction, VT, USA). Anti-MPO antibody was purchased from Santa Cruz (Dallas, TX, USA). Ficoll ${ }^{\circledR}$ Paque Plus was purchased from GE Healthcare (Chicago, IL, USA). Roswell Park Memorial Institute (RPMI) Media 1640 was purchased from Gibco (Thermo Fisher Scientific, Waltham, MA, USA). BioCoat Cellware poly-L-lysine $12 \mathrm{~mm}$ coverslips were purchased from Becton Dickinson (La Jolla, CA, USA). Goat anti-rabbit lgG $(\mathrm{H}+\mathrm{L})$ secondary antibody, Alexa Fluor ${ }^{\circledR} 488$, was purchased from Invitrogen (Thermo Fisher Scientific, Waltham, MA, USA).

\subsection{Isolation of neutrophils}

Heparinized whole blood was withdrawn from fasting, non-smoking, apparently healthy volunteers, who had not received any anti-platelet and anti-inflammatory drugs for at least two weeks prior to venipuncture. The whole blood was diluted with normal saline, it was care- fully overlayed on a Histopaque ${ }^{\oplus}-1119$ and Ficoll ${ }^{\circledast}$ Paque Plus gradient and then it was centrifuged at $700 \times g$ for 30 min, at room temperature (RT). The polymorphonuclear leukocyte (PMN) zone was aspirated, diluted with 12.5 $\mathrm{mM}$ phosphate-buffered saline (PBS) and centrifuged at $200 \times g$ for $10 \mathrm{~min}$, at RT. Finally, the PMN pellet, enriched in neutrophils, was re-suspended with RPMI Media 1640, supplemented with $2 \% \mathrm{HI}$ human serum.

\subsection{Preparation of platelet-rich plasma}

Platelet-rich plasma (PRP), autologous to the neutrophils, was prepared as previously described. ${ }^{9}$ Briefly, whole blood was centrifuged at $126 \times g$ for $15 \mathrm{~min}$, at RT. The upper two-thirds of the PRP layer were aspirated and the remaining PRP was further centrifuged at $1500 \times g$ for $20 \mathrm{~min}$, at RT, to obtain platelet-poor plasma (PPP). The number of platelets in the PRP was counted using a hemocytometer and it was adjusted to $2.5 \times 10^{5} / \mu \mathrm{L}$ with PPP.

\subsection{NETs generation and visualization}

Neutrophils $\left(2 \times 10^{5}\right)$ were added to 24 -well plates, in which poly-L-lysine coverslips were pre-placed, and incubated for $1 \mathrm{~h}$ at $37^{\circ} \mathrm{C}$ and $5 \% \mathrm{CO}_{2}$, in order to allow the cells to bind to the coverslips.

The effect of coagulation factors and various classic platelet agonists on NETs generation was investigated. We tested FXa (25-50 nM), thrombin (0.2-8 U/mL), ADP (5-100 $\mu \mathrm{M}), \mathrm{AA}(0.1-0.5 \mathrm{mM})$, collagen $(5-15 \mu \mathrm{g} / \mathrm{mL})$ and PAR-4 activating peptide (sequence AYPGKF-NH $\mathrm{N}_{2} ; 100$ $\mu \mathrm{M})$. In addition, we studied the effect of a selective, PAR-2 activating peptide (sequence SLIGRL-NH ${ }_{2} ; 10-100$ $\mu \mathrm{M}$ ) on the formation of NETs, since PAR-2 is expressed on neutrophils. ${ }^{10}$ PMA, a widely used NET inducer, was used as a positive control for induction of NETosis at a concentration range of 25-100 nM.

In other experiments, PRP was activated with $20 \mu \mathrm{M}$ ADP at $37^{\circ} \mathrm{C}$ for $5 \mathrm{~min}$, under 1200 RPM stirring conditions, in a Chrono-Log Model 700 Lumi-Aggregometer (ChronoLog Corporation, Philadelphia, PA, USA) and the total suspension of the activated PRP was then immediately transferred to neutrophils, at a physiological ratio of 150 $\times 10^{3}$ platelets $/ 6 \times 10^{3}$ neutrophils. Alternatively, PRP was added to neutrophils, at the above ratio, and activated in situ with $50 \mu \mathrm{M}$ ADP.

In all the above experimental conditions, neutrophils were incubated for $3.5 \mathrm{~h}$ at $37^{\circ} \mathrm{C}$ and $5 \% \mathrm{CO}_{2}$, to produce NETs. Afterwards, the cells and NETs were fixed and then an immunofluorescence protocol was followed. According to this protocol, neutrophils and NETs were incubated with an anti-MPO primary antibody and the goat anti-rabbit $\lg \mathrm{G}(\mathrm{H}+\mathrm{L})$ secondary antibody, Alexa Fluor $^{\circledR} 488$, for MPO 
visualization. In addition, neutrophils and NETs were incubated with $200 \mathrm{ng} / \mathrm{mL}$ DAPI, for DNA visualization. NETs were imaged with an Olympus BX41 fluorescence microscope and with a Leica TCS SP5 confocal microscope, with a HC PL APO CS $63 \times$ oil lens and the LAS AF software. The number of cells that release NETs was counted, and the percentage of NET-releasing neutrophils was obtained from 5 representative optical fields.

\section{RESULTS AND DISCUSSION}

The coagulation factors FXa and thrombin were tested at a range of concentrations of 25-50 nM and 0.2-8 U/mL, respectively. Even at the maximal concentration tested, $\mathrm{FXa}$ or thrombin did not induce substantial NETosis (Figure 1).

To study the effect of activated platelets and therefore the effect of platelet - neutrophil cell to cell interaction or the effect of platelet-secreted mediators, we first studied the effect of various platelet agonists on NETosis, in a wide variety of concentrations. Among all agonists tested, only PMA and AA induced robust NETosis (Figure 2A). The threshold concentration of the above agonists that induced the maximum of NETs production, were $25 \mathrm{nM}$ for PMA and $0.25 \mathrm{mM}$ for AA. All other agonists, i.e. ADP, collagen, PAR-4 and PAR-2 agonists, even at the maximum concentration tested, did not induce substantial NETosis (Figure 2A). Representative confocal microscopy images of untreated neutrophils, as well as PMA- and AA-induced NETosis, at the maximum concentration tested, are shown in Figure 2B.

We next evaluated whether activated platelets can induce NETosis. To activate platelets in situ, i.e. in the presence of neutrophils, we have chosen a platelet agonist, among those tested in the present study, that was inactive towards NETs production by neutrophils. Among these

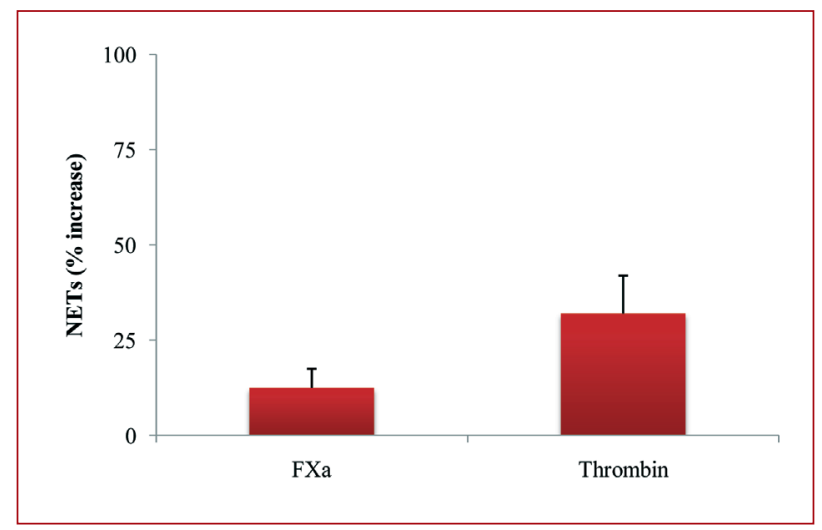

FIGURE 1. Effect of coagulation factors on the formation of NETs. The bar graph depicts NETosis induced by $50 \mathrm{nM} \mathrm{FXa}$ and $8 \mathrm{U} / \mathrm{mL}$ thrombin. The above results represent the mean \pm SD of at least 4 different neutrophil preparations.

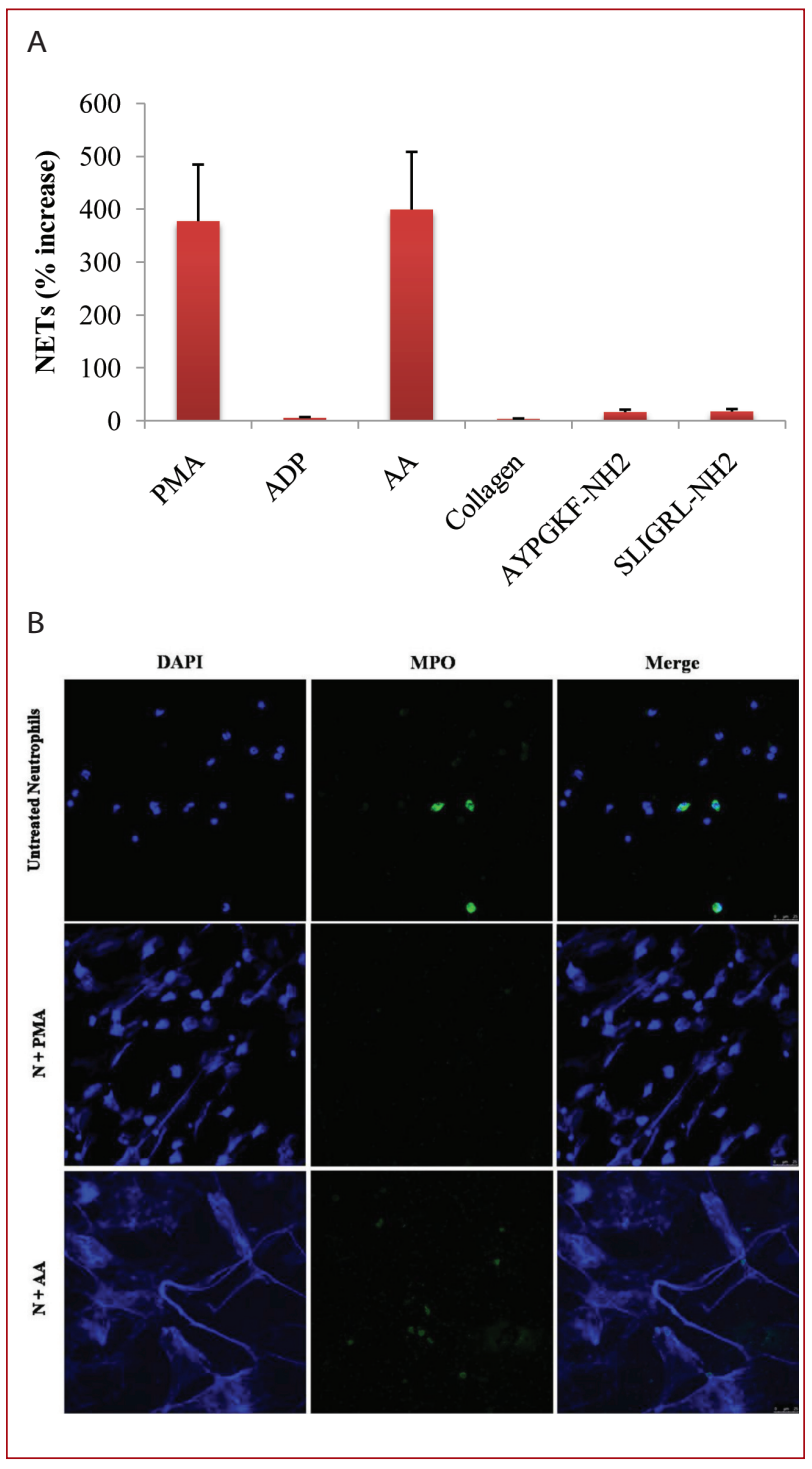

FIGURE 2. A. Effect of various agonists on the formation of NETs. The bar graph depicts NETosis induced by $25 \mathrm{nM}$ PMA, $100 \mu \mathrm{M}$ ADP, $0.25 \mathrm{mM} \mathrm{AA}, 15 \mu \mathrm{g} / \mathrm{mL}$ collagen, $100 \mu \mathrm{M}$ AYPGKF-NH $\mathrm{N}_{2}$ and $100 \mu \mathrm{M}$ SLIGRL-NH $\mathrm{N}_{2}$. The above results represent the mean \pm SD of at least 4 different neutrophil preparations. B. Representative confocal microscopy images of untreated neutrophils, as well as NETs from neutrophils treated with $25 \mathrm{nM}$ PMA or $0.25 \mathrm{mM} \mathrm{AA}$. Neutrophils and NETs were stained for myeloperoxidase (MPO) and DNA with DAPI. Magnification 63×. Abbreviation: N; neutrophils.

agonists we have chosen ADP since its platelet receptor $\mathrm{P} 2 \mathrm{Y} 12$, is the main target of antiplatelet therapy used currently in the clinical practice. ${ }^{11}$ ADP cannot induce neutrophil activation and NETosis, since neutrophils do not express functional $\mathrm{P} 2 \mathrm{Y} 12$ receptor. ${ }^{12}$ We first investigated whether the total suspension of platelets activated with ADP in an aggregometer could induce NETosis. As expected, ADP, at the concentration of $20 \mu \mathrm{M}$, induced a full platelet aggregatory response when added to the 
PRP in the aggregometer (Figure 3A). The total suspension of the ADP-activated PRP induced a significant NETosis when incubated with the neutrophil suspension for $3.5 \mathrm{~h}$ as compared with resting PRP (Figure 3B). Similarly, when the PRP was activated with ADP in situ, in the presence of neutrophils, it induced NETs production, which was not observed in the absence of ADP (Figure 3C). Representative confocal microscopy images of neutrophils in the presence of untreated PRP, as well as in the presence of PRP, either pre-activated in the aggregometer or activated in situ with ADP, are shown in Figure 3D.

The present study demonstrates that the coagulation factors FXa and thrombin, as well as the platelet agonists ADP, collagen and PAR-4 agonist, do not induce NETs production. This is possibly due to the absence of the expression of their corresponding functional receptors on neutrophils, or due to the fact that these receptors may be linked to other neutrophil functions, such as chemotaxis, but not to NET formation. ${ }^{12-14}$ It has been suggested that PAR-2 is expressed in neutrophils. ${ }^{10}$ However, in the present study we showed that the PAR-2 activating peptide does not cause substantial NETosis. This could be possibly due to the fact that this receptor may be linked to other neutrophil functions but not to NET formation, or this receptor may be not functional in neutrophils.

By contrast the platelet agonist $A A$, induced robust NETosis, comparable in magnitude to PMA, the potent neutrophil activator and inducer of NETs production. In platelets, AA is physiologically hydrolyzed from the membrane bilayer phospholipids, or taken up by them when added in vitro, to form thromboxane $A_{2}$ via the cyclooxygenase-1 pathway. ${ }^{15}$ In neutrophils, AA is metabolized via the lipoxygenase-1 pathway, which among other active metabolites, it leads to the formation of leukotriene $B_{4}{ }^{16}$
A

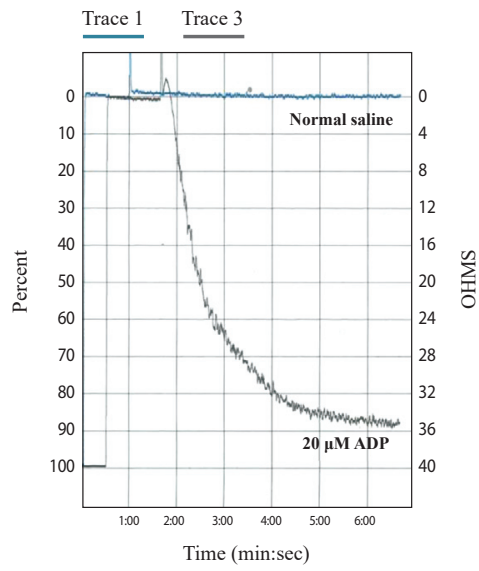

C

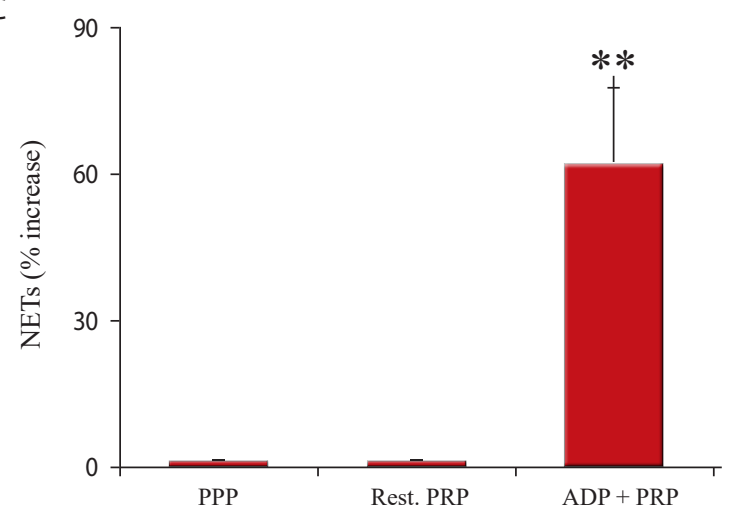

B

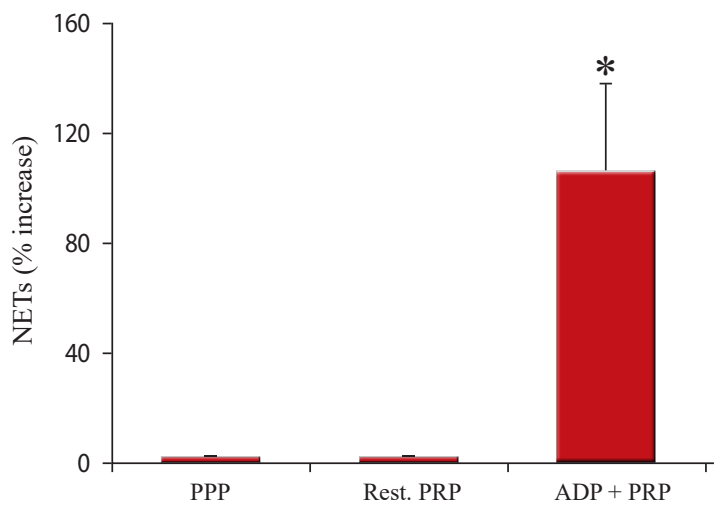

D

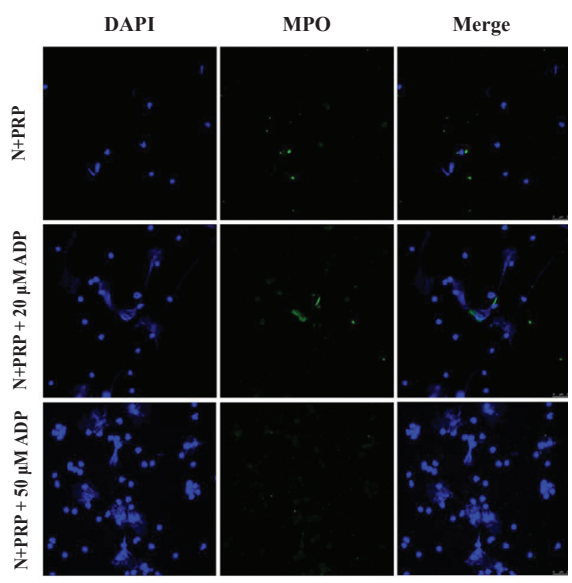

FIGURE 3. A. Representative aggregation curve of the response of PRP, activated with $20 \mu \mathrm{M}$ ADP. B. Effect of PRP, treated with $20 \mu \mathrm{M}$ ADP in an aggregometer, on the formation of NETs ( ${ }^{*} p=0.03$, compared with either PPP or untreated [resting; Rest.] PRP). C. Effect of PRP, treated with $50 \mu \mathrm{M}$ ADP in situ, on the formation of NETs ( ${ }^{* *} p=0.011$, compared with either PPP or untreated [resting; Rest.] PRP). D. Representative confocal microscopy images of neutrophils in the presence of untreated PRP, as well as in the presence of PRP, either pre-activated in the aggregometer or activated in situ with ADP. Neutrophils and NETs were stained for myeloperoxidase (MPO) and DNA with DAPI. Magnification 63×. Abbreviation: N; neutrophils. 
that could be the main metabolite responsible for the AA-induced NETosis.

An important observation of the present study is that in the presence of platelets, ADP can induce NETosis even though it is inactive when added directly to neutrophils. This finding is consistent with our observation that the suspension of platelets activated with ADP, also induces NETosis. These results suggest that activated platelets, through cell to cell interaction with neutrophils or by secreting mediators such as $\mathrm{P}$-selectin or AA or by shedding platelet microparticles could potently induce the production of NETs.

In conclusion, the present study demonstrates that activated platelets induce NETs production thus providing an additional mechanism through which platelets contribute to various pathophysiological conditions in which NETs are implicated, such as inflammation and thrombosis. This new finding may be clinically significant in patients receiving antiplatelet therapy with the platelet P2Y12 antagonists clopidogrel or ticagrelor. The whole spectrum of platelet mediators that are implicated in NETosis needs to be further investigated.

\section{Acknowledgments}

The present research has been co-financed by the Operational Program "Human Resources Development, Education and Lifelong Learning" and is co-financed by the European Union (European Social Fund) and Greek national funds [grant number (MIS) 5006031].

\section{Conflict of interest}

The authors report no conflicts of interest.

\title{
ПЕРІАНЧН
}

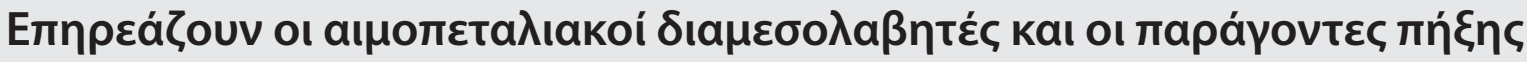

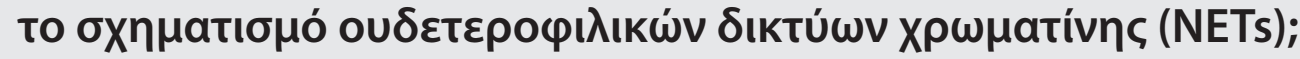

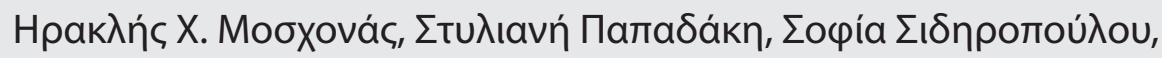

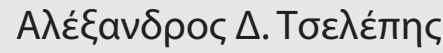

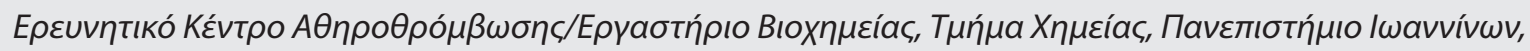 \\ I $\omega a ́ v v i v a, E \lambda \lambda a ́ \delta a$
}

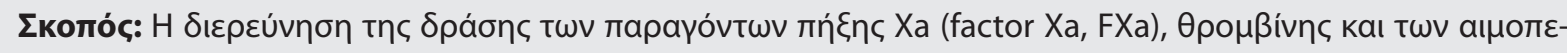

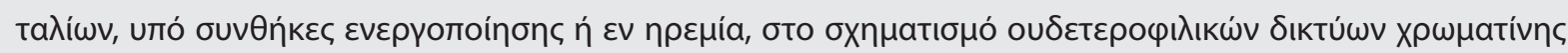

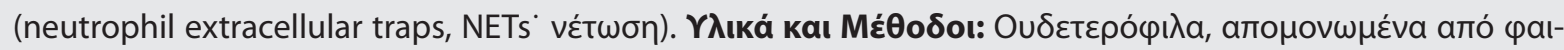

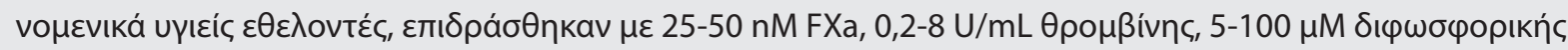

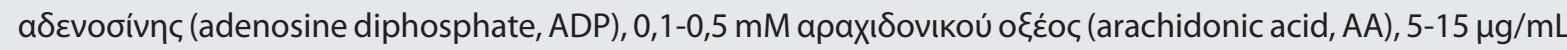

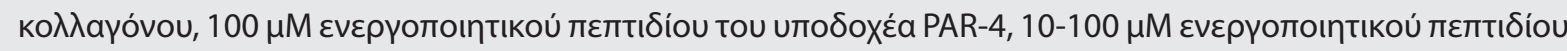

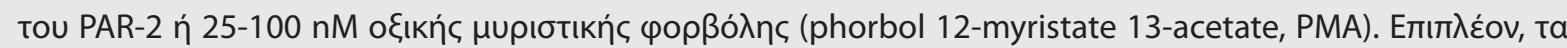

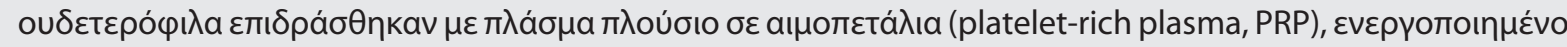

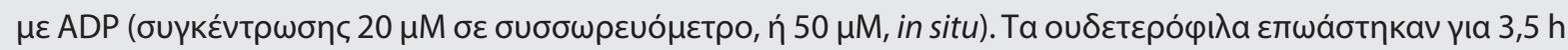

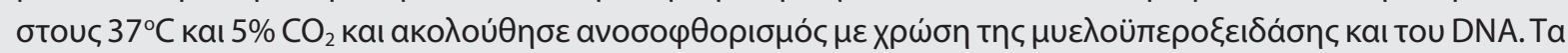

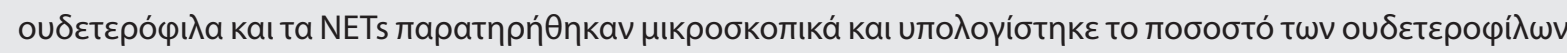

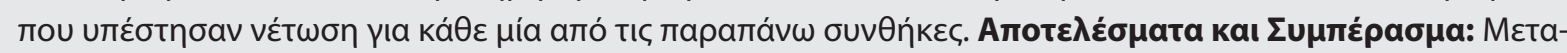

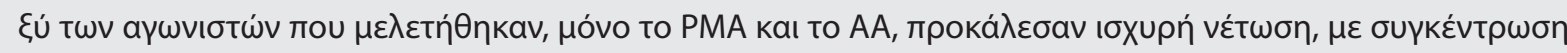

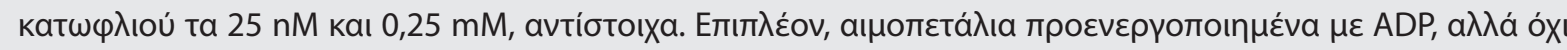

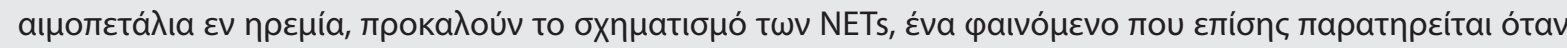

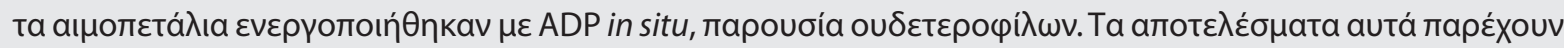

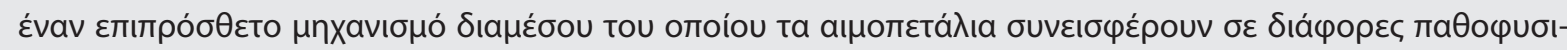

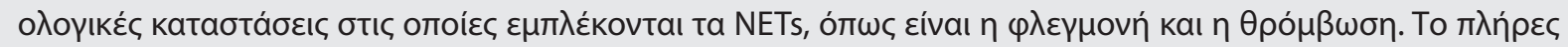

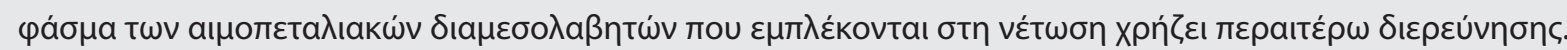

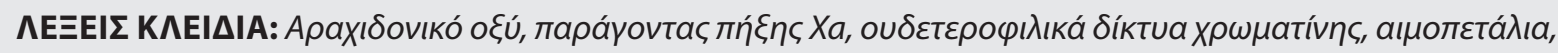
$\theta \rho o \mu \beta i v \eta$ 


\section{REFERENCES}

1. Brinkmann V, Reichard U, Goosmann C, Fauler B, Uhlemann Y, Weiss DS, et al., Neutrophil extracellular traps kill bacteria. Science. 2004 Mar;303(5663):1532-5.

2. Cools-Lartigue J, Spicer J, Najmeh S, Ferri L. Neutrophil extracellular traps in cancer progression. Cell Mol Life Sci. 2014 Nov;71(21):4179-94.

3. Wong SL, Demers M, Martinod K, Gallant M, Wang Y, Goldfine $A B$, et al, Diabetes primes neutrophils to undergo NETosis, which impairs wound healing. Nat Med. 2015 Jul;21(7):815-9.

4. Pietronigro EC, Della Bianca V, Zenaro E, Constantin G. NETosis in Alzheimer's Disease. Front Immunol. 2017 Mar;8:211.

5. Moschonas IC, AD Tselepis. The pathway of neutrophil extracellular traps towards atherosclerosis and thrombosis. Atherosclerosis. 2019 Sep;288:9-16.

6. Moschonas IC, Goudevenos JA, Tselepis AD. Proteaseactivated receptor-1 antagonists in long-term antiplatelet therapy. Current state of evidence and future perspectives. Int J Cardiol. 2015 Apr;185:9-18.

7. Papadaki S, Tselepis AD. Nonhemostatic Activities of Factor Xa: Are There Pleiotropic Effects of Anti-FXa Direct Oral Anticoagulants? Angiology. 2019 Nov;70(10): 896-907.

8. Kalantzi KI, Dimitriou AA, Milionis HJ, Goudevenos IA, Tselepis AD. Clopidogrel differentially affects plateletmediated thrombosis and inflammatory response in patients with acute coronary syndromes. JThromb Haemost. 2011 Apr;9(4):875-8.

9. Tsoumani ME, Tatsidou PT, Ntalas IV, Goudevenos JA,
Tselepis AD. Dynamic platelet adhesion in patients with an acute coronary syndrome: The effect of antiplatelet therapy. Platelets. 2016 Dec;27(8):812-20.

10. Bock A, Tucker N, Kelher MR, Khan SY, Gonzalez E, Wohlauer $\mathrm{M}$, et al. Alpha-Enolase Causes Proinflammatory Activation of Pulmonary Microvascular Endothelial Cells and Primes Neutrophils Through Plasmin Activation of Protease-Activated Receptor 2. Shock. 2015 Aug;44(2):137-42.

11. Tsoumani ME, Tselepis AD. Antiplatelet Agents and Anticoagulants: From Pharmacology to Clinical Practice. Curr Pharm Des. 2017;23(9):1279-93.

12. Le Duc D, Schulz A, Lede V, Schulze A, Thor D, Brüser A, et al. P2Y Receptors in Immune Response and Inflammation. Adv Immunol. 2017;136:85-121.

13. Kahn ML, Nakanishi-Matsui M, Shapiro MJ, Ishihara H, Coughlin SR. Protease-activated receptors 1 and 4 mediate activation of human platelets by thrombin. J Clin Invest. 1999 Mar;103(6):879-87.

14. Senior RM, Hinek A, Griffin GL, Pipoly DJ, Crouch EC, Mecham RP. Neutrophils show chemotaxis to type IV collagen and its $7 \mathrm{~S}$ domain and contain a $67 \mathrm{kD}$ type IV collagen binding protein with lectin properties. Am J Respir Cell Mol Biol. 1989 Dec;1(6):479-87.

15. Patrono C, Rocca B. Measurement of Thromboxane Biosynthesis in Health and Disease. Front Pharmacol. 2019 Oct; 10:1244.

16. Raulf M, Konig W. Modulation of leukotriene generation from human polymorphonuclear granulocytes by polychlorinated biphenyls (PCB). Immunology. 1991 Aug;73(4):485-90. 